\title{
Experience of Intraoperative Recurrent Laryngeal Nerve monitoring in a Single Center-normative Recurrent Laryngeal Nerve Electromyographic Data
}

\author{
${ }^{1}$ Gilbert Soh, ${ }^{2}$ James Wai Kit Lee, ${ }^{3}$ Oh Han Boon, ${ }^{4}$ Tan Wee Boon, ${ }^{5}$ Rajeev Parameswaran, ${ }^{6}$ Ngiam Kee Yuan
}

\begin{abstract}
Introduction: Injury to the recurrent laryngeal nerve (RLN) remains a significant morbidity during thyroid and parathyroid surgery. The aim of this study is to elucidate normative RLN electromyographic (EMG) parameters.
\end{abstract}

Materials and methods: This is a retrospective cohort study of patients who underwent Intraoperative neuromonitoring during thyroid and parathyroid surgery from February 2014 to March 2015. The inomed C2 NerveMonitor was used. We recorded the stimulation current, amplitude, and latency of the RLN before and after nerve dissection. We also observed the number of patients who had hoarse voice after surgery.

Results: A total of 46 patients (14 male, 32 female) averaging 51 years old in age (20-77 years) were analyzed. The most commonly performed surgical procedure was total thyroidectomy $(53.2 \%)$.

The median stimulation current for both the right and left $R L N$ was $0.500 \mathrm{~mA}$. The median amplitude for the left $R L N$ was $1.060 \mathrm{mV}$ and greater than that for the right RLN $(0.930 \mathrm{mV})$ $(p=0.30)$. The median latency for the right RLN and left RLN was 2.40 ms with no difference between the sides. $(p=0.58)$.

Post dissection, the right RLN amplitude remained identical whereasthe left RLN amplitude decreased. Latencies of both RLNs decreased although the difference was not significant. Nature of pathology and site of surgery did not influence RLN latency and amplitude. No patients had hoarse voice.

Conclusion: This study highlights the normative EMG parameters for bilateral RLN nerve stimulation in an Asian population. No significant difference was noted in both pre- and postdissection RLN EMG parameters.

Keywords: Amplitude, Intraoperative nerve monitoring, Latency, Normative electromyographic values, Recurrent laryngeal nerve palsy, Stimulation current.

How to cite this article: Soh G, Lee JWK, Boon OH, Boon TW, Parameswaran R, Yuan NK. Experience of Intraoperative Recurrent Laryngeal Nerve monitoring in a Single Centernormative Recurrent Laryngeal Nerve Electromyographic Data. World J Endoc Surg 2017;9(1):1-6.

\footnotetext{
${ }^{1}$ Student (Final Year), ${ }^{2}$ Resident, ${ }^{3}$ Registrar, ${ }^{4}$ Associate Consultant, ${ }^{5,6}$ Consultant

${ }^{1-6}$ Department of General Surgery, National University Hospital Singapore

Corresponding Author: Ngiam Kee Yuan, Consultant Department of General Surgery, National University Hospital Singapore, Phone: +6596600471, e-mail: Kee_yuan_ngiam@ nuhs.edu.sg
}

\section{Source of support: Nil}

Conflict of interest: None

\section{INTRODUCTION}

Injury to the recurrent laryngeal nerve (RLN) remains a significant morbidity during thyroid, parathyroid and head and neck surgery. The use of intraoperative neuro monitoring is a useful adjunct to reduce or prevent RLN injury but requires experience in the appropriate use and interpretation of its signals to ensure uniform results it is application during surgery. It is possible to differentiate between the RLN, vagus nerve and external branch of the superior laryngeal nerve from latency information found on the neuromonitor trace and thence permits the identification of anatomical variants of these nerves. Furthermore, alterations in neuro monitoring traces throughout surgery is appropriate within limits, and understanding this allows the operator to determine the tolerances of nerve dissection during surgery. The aim of this study is to elucidate normative RLN electromyographic (EMG) parameters during the use of the neuromonitor in head and neck surgery.

\section{Anatomy of the Recurrent Laryngeal Nerve}

The thyroid gland is composed of two lobes connected by an isthmus that lies on the trachea at the level of the second tracheal ring. The right recurrent laryngeal nerve (RLN) arises from the vagus nerve, loops around the right subclavian artery and enters the larynx behind the cricothyroid muscle. It innervates all the intrinsic laryngeal muscles except the cricothyroid. The left RLN originates from the left vagus nerve, loops around the arch of the aorta, and ascends in the trachea-esophageal groove. It innervates similar muscles as the right RLN. ${ }^{1,2}$ Damage to the RLNs causes difficulty in breathing and phonation. Injury to RLN during neck dissection remains a significant cause of morbidity during neck dissection. Injury rates are estimated to be $2-5 \%$. $^{3,4}$ Recurrent laryngeal nerve injury is also a leading cause of litigation for thyroid surgeons. 5,6

\section{ROLE OF INTRAOPERATIVE NEUROMONITORING}

Intraoperative neuromonitoring (IONM) is widely used as an adjunct during thyroid surgery, aiming to help 
surgeons identify the RLN and dissect it. ${ }^{7-13}$ Intraoperative neuromonitoring can also help to predict and prognosticate the degree of RLN nerve injury. ${ }^{14-17}$ Previous studies of normative EMG data have been done in American and European populations. ${ }^{18-20}$ Hopefully, by defining the normative RLN monitoring parameters, we can predict the degree of nerve injury following which nerve monitoring signals may be altered or lost. ${ }^{21-23}$ Our study aims to establish the normal RLN EMG data in an Asian population.

\section{MATERIALS AND METHODS}

This is a retrospective cohort study of 46 patients who underwent thyroid or parathyroid surgery at a tertiary referral center between 2014 and 2015. Patients with preexisting vocal cord palsy were excluded. Intraoperative neuromonitoring was applied accordingly to a strict study protocol applying a standardized setup using the inomed C2 NerveMonitorn ${ }^{\circledR}$ (inomed Medizintechnik $\mathrm{GmbH}$, Emmendingen, Germany) and a noninvasive endotracheal tube surface electrode monitoring the thyroid arytenoid muscle. This was adapted from the protocol recommended by Poveda et al. ${ }^{24,25}$ Vocal cord mobility was visualized and recorded at the time of tracheal intubation. Anesthesia was induced using only short acting nondepolarizing paralytic agents (succinylcholine at $2-2.5 \mathrm{mg} / \mathrm{kg}$ ), which wore off within 10 minutes and thus did not affect subsequent neuromuscular function.

\section{Events Studied}

Qualitative parameters, such as hoarseness of voice were observed at the following time points: Immediately post operation, at postoperative day 1 , and at 2 weeks follow-up. Intraoperative neuromonitoring quantitative parameters of the RLN assessed were stimulation current, latency and amplitude. Both the pre and post-dissection values were recorded.

The definitions of nerve latency and amplitude are stated in Table 1, with reference to Phelan et al's definitions. $^{18}$

The stimulation current was set at the range of $0.50 \mathrm{~mA}$, after taking into account the manufacturers' recommendations and later modulated through direct

Table 1: Definitions of quantitative parameters of the RLN latency and amplitude assessed during IONM

\begin{tabular}{lll}
\hline Parameter & Units & Definition \\
\hline Latency & Milliseconds $(\mathrm{ms})$ & $\begin{array}{l}\text { Time between the stimulation } \\
\text { artifact and onset of EMG } \\
\text { activity }\end{array}$ \\
Amplitude & Millivolts $(\mathrm{mV})$ & $\begin{array}{l}\text { Maximum deflection from the } \\
\text { baseline of the EMG wave }\end{array}$ \\
\hline
\end{tabular}

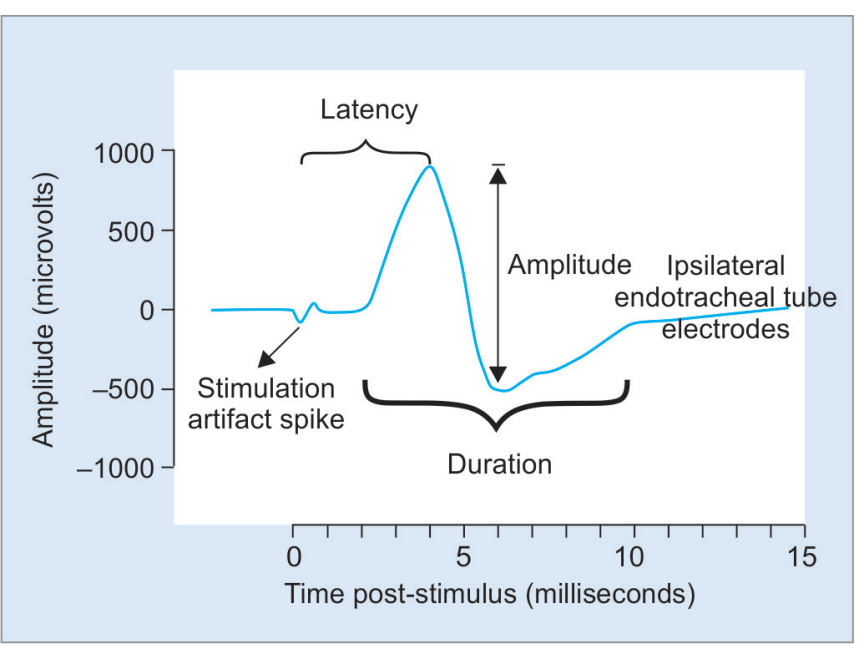

Graph 1: Stimulation artifact. Reproduced with permission from The Laryngoscope journal: "Intraoperative monitoring: Normative range associated with normal postoperative glottis function" by Caragacianu et al

stimulation of the nerve and nonnerve structures. In addition, Faden $\mathrm{et}^{\mathrm{al}}{ }^{26}$ has found that $0.50 \mathrm{~mA}$ of stimulation current optimizes sensitivity and specificity of nerve monitoring.

Discrimination between stimulation artifacts and evoked responses was established by observing at the EMG wave configurations. Stimulation artifact had a sharp and early spike whereas neural EMG activity resulted in broader and later deformation of baseline with a typical biphasic course (Graph 1). ${ }^{19}$ The protocol for nerve stimulation mandates the RLN to be stimulated at where it exits the thoracic cavity and at the tracheoesophageal groove where it crosses the inferior thyroid artery. Stimulation of the nerve was done $4 \mathrm{~cm}$ prior to its entry point into the larynx behind the cricothyroid muscle.

The results of RLN amplitudes and latency were compared between the right and left sides, before and after dissection, males and, females and in cases of benign vs malignant disease. The magnitude difference in the amplitude/latency is defined as post-dissection amplitude/latency minus pre-dissection amplitude/latency.

Statistical analysis was performed using the Statistical Package for the Social Sciences (SPSS) (version 19.0, SPSS Inc., Chicago, Illinois). Pre- and post-dissection stimulation current, amplitude, and latency were compared using Wilcoxon signed rank test. Mann-Whitney $\mathrm{U}$ test was done to test the null hypothesis against the various alternative hypotheses. Two-tailed $\mathrm{p}<0.05$ was considered statistically significant.

\section{RESULTS}

The mean age of the patients was 51 years old ( $20-77$ years). Fourteen of the patients were male and 32 female. There were 25 cases of total thyroidectomy (55\%), 13 
Table 2: Comparing the right and left RLN pre-dissection stimulation current, amplitude, and latency

\begin{tabular}{llll}
\hline & $\begin{array}{l}\text { Right } \\
\text { RLN }\end{array}$ & Left & \\
& $R L N$ & $p$-value \\
\hline $\begin{array}{l}\text { Median pre-dissection stimulation } \\
\text { current/mA }\end{array}$ & 0.500 & 0.500 & $\mathrm{NS}$ \\
Median pre-dissection amplitude/ $\mu \mathrm{V}$ & 930 & 1060 & $\mathrm{NS}$ \\
Median pre-dissection latency/ms & 2.40 & 2.40 & $\mathrm{NS}$ \\
\hline NS: Not significant & & &
\end{tabular}

hemithyroidectomies (28\%), 7 parathyroidectomies $(15 \%)$, and 1 neck re-exploration (2\%). The mean operation duration was 80.9 minutes.

Baseline IONM stimulation values and nerve monitoring parameters were similar between the right and left RLN (Table 2).

\section{Intraoperative neuromonitoring Parameters before and after Dissection}

The right median RLN amplitude remained identical before and after dissection whereas the left median RLN amplitude decreased by $150 \mu \mathrm{V}$. Both the right and left median RLN latency decreased after dissection (Table 3).

Table 3: Comparing RLN amplitude and latency pre- and post-dissection on the left and right side

\begin{tabular}{llll}
\hline & \multicolumn{2}{c}{ Amplitude } & \\
\cline { 2 - 3 } & $\begin{array}{l}\text { Right RLN } \\
\text { amplitude/mV }\end{array}$ & $\begin{array}{l}\text { Left } R L N \\
\text { amplitude/mV }\end{array}$ & p-value \\
\hline Before dissection & $0.93(0.17-3.27)$ & $1.06(0.44-3.23)$ & NS \\
After dissection & $0.93(0.2-3.53)$ & $0.91(0.29-4.15)$ & NS \\
\hline & \multicolumn{2}{c}{ Latency } & \\
\cline { 2 - 3 } & Right RLN & Left RLN & \\
& latency/ms & latency/ms & \\
\hline Before dissection & $2.4(1.60-5.40)$ & $2.4(1.35-3.30)$ & \\
After dissection & $2.2(1.4-5.15)$ & $2.35(1.35-3.90)$ & \\
\hline NS: Not significant & &
\end{tabular}

\section{Intraoperative neuromonitoring Parameters according to Gender}

For the right RLN, females had higher pre-dissection median amplitude (Table 4). However, females had lower right RLN post-dissection median amplitudes. For the left RLN, females had lower pre- and post-dissection median amplitudes. None of the comparisons between male and female median amplitudes was statistically significant.

Females had a lower median RLN latency for both the right and left RLN. All the comparisons were not statistically significant except for the comparison between males and females for the left pre-dissection median latency. Females had a statistically significant $(p=0.04)$ lower pre-dissection median latency than males.

\section{Intraoperative neuromonitoring Parameters according to the Nature of Disease: Benign/ Malignant}

In general, benign lesions had lower median RLN amplitudes compared to malignant lesions. Between benign vs malignant lesions, the amplitudes were not significantly different between both the pre- and post-dissection median amplitude bilaterally (Table 5). This was irrespective of the size of the lesion, the presence of a concomitant neck dissection and stage of the thyroid cancer.

For the right RLN, benign lesions had a higher median latency compared to malignant lesions. For the left RLN, malignant lesions had higher median latency than benign lesions. Between benign vs malignant lesions, the latencies were not significantly different between both the pre- and post-dissection median latencies bilaterally.

The magnitude difference between pre- and postdissection amplitude is $0.05 \mathrm{mV}$ for the right and left RLN. The difference between the magnitude differences

Table 4: Comparing the quantitative IONM parameters between female and male patients

\begin{tabular}{|c|c|c|c|c|c|c|}
\hline & \multicolumn{3}{|c|}{ Right RLN } & \multicolumn{3}{|c|}{ Left RLN } \\
\hline & Male & Female & $p$-value & Male & Female & $p$-value \\
\hline Pre-dissection median amplitude/ $\mu \mathrm{V}$ & 900 & 940 & NS & 1190 & 990 & NS \\
\hline Post-dissection median amplitude/ $\mu \mathrm{V}$ & 930 & 920 & NS & 1070 & 795 & NS \\
\hline Pre-dissection median latency/ms & 2.400 & 2.300 & NS & 2.600 & 2.250 & 0.04 \\
\hline Post-dissection median latency/ms & 2.500 & 2.100 & NS & 2.450 & 2.275 & NS \\
\hline
\end{tabular}
NS: Not significant

Table 5: Comparing the quantitative parameters of the benign vs malignant lesions

\begin{tabular}{|c|c|c|c|c|c|c|}
\hline & \multicolumn{3}{|c|}{ Right RLN } & \multicolumn{3}{|c|}{ Left RLN } \\
\hline & Malignant & Benign & $p$-value & Malignant & Benign & $p$-value \\
\hline Pre-dissection median amplitude/ $\mu \mathrm{V}$ & 950 & 915 & NS & 1140 & 880 & NS \\
\hline Post-dissection median amplitude/ $\mu \mathrm{V}$ & 1020 & 875 & NS & 1020 & 835 & NS \\
\hline Pre-dissection median latency/ms & 2.200 & 2.400 & NS & 2.425 & 2.400 & NS \\
\hline Post- dissection median latency/ms & 2.150 & 2.350 & NS & 2.450 & 2.250 & NS \\
\hline
\end{tabular}

NS: Not significant 
for each side is not statistically significant. The magnitude difference between pre- and post-dissection latency is $-0.1 \mathrm{~ms}$ for the right RLN and $0.02 \mathrm{~ms}$ for the left RLN. The difference between the magnitude differences for each side is not statistically significant.

None of the patients developed hoarseness of voice postoperatively.

\section{DISCUSSION}

This is the first study that examines the EMG characteristics of RLNs before and after dissection in an Asian population. We hypothesized that there could be a difference in the EMG data between the right and left RLNs, between benign and malignant thyroid disease and between male and female patients.

The results showed that there was no significant difference between pre- and post-dissection amplitude and latency in all categories of comparison: Male $v s$ female, benign $v s$ malignant disease, and side of surgery. This is consistent with published studies by Lorenz et al ${ }^{19}$ Phelan et $\mathrm{al}^{18}$, and Caragacianu et $\mathrm{al}^{20}$ (Table 6).

There are, however, some differences between our study and the other three studies. First, our study found that there was a small significant difference in the leftsided pre-dissection latency between males and females. This is unlikely to be clinically significant. Second, Lorenz et al ${ }^{19}$ found that females had a higher median RLN amplitude than males for both the RLNs. In our study, females had lower median amplitude on the left RLN but higher median amplitudes on the right RLN. Third, Lorenz et $\mathrm{al}^{19}$ found a significant difference of median RLN amplitude between males and females $(p<0.05)$ but our study did not observe the same difference and this is consistent with the study by Phelan et al ${ }^{18}$ (Table 7).
The differences seen in this study compared with others are probably due to technical differences in the IONM systems; variation of amplitude measurement due to factors, such as contact pressure, obscuration of the nerve by blood or other fluids and amount of overlying tissue between the stimulator probe and the nerve.

In our study, we observed that the amplitude change before and after dissection remained the same on the right RLN and decreased on the left RLN. This is contrary to the finding by Phelan et $\mathrm{al}_{1}^{18}$ Caragacianu et $\mathrm{al}^{20}$ who reported an increase in RLN amplitude bilaterally preand post-dissection. This could be due to physiological differences between Asian and Caucasian populations and different techniques of nerve stimulation.

We initially hypothesized that the nature of the lesion would affect the risk of injury and hence, the conduction characteristics of the RLN pre- and post-dissection. However, with regards to the nature of the lesion (benign/malignant) on quantitative IONM parameters, there is no statistical difference in RLN amplitude/ latency when comparing benign with malignant lesions. Lorenz et $\mathrm{l}^{19}$ also concluded that benign/malignant thyroid disease did not affect IONM parameters.

Lastly, we hypothesized that the pre-dissection amplitude and latency of the right recurrently laryngeal nerve would change more after dissection as the course of the right RLN is more oblique and is hence, more prone to injury during surgery. However, our data demonstrated no statistically significant difference in magnitude of change of pre-dissection RLN amplitude/latency when comparing the right and left RLN.

Our study is limited and it is a retrospective study with possible selection biases. In addition, the sample size was small with 46 patients, hence we do not have any EMG data on any damaged nerves. However, in this

Table 6: Comparing the median amplitude and latency of all four studies of RLN monitoring data on human subjects

\begin{tabular}{|c|c|c|c|c|c|c|c|}
\hline & \multicolumn{2}{|c|}{ Lorenz et al ${ }^{19}$} & \multicolumn{2}{|c|}{ Phelan et al ${ }^{18}$} & \multirow[b]{2}{*}{ Caragacianu et al20 } & \multicolumn{2}{|c|}{ Our study } \\
\hline & Right & Left & Right & Left & & Right & Left \\
\hline Median amplitude/ $/ \mathrm{V}$ & 623 & 719 & $783^{b}$ & $604^{\mathrm{b}}$ & $898^{\mathrm{b}, \mathrm{c}}$ & 930 & 1060 \\
\hline Median latency/ms & 2.73 & 2.73 & 3.10 & 4.25 & - & 2.40 & 2.40 \\
\hline
\end{tabular}

${ }^{\mathrm{b}}$ Mean amplitude was stated in the study by Phelan et $\mathrm{al}^{18}$ and Caragacianu et al ${ }^{20} ;{ }^{\mathrm{C}} \mathrm{Caragacianu}$ et al ${ }^{20}$ did not quantify the amplitude of the left and right RLN separately

Table 7: Comparing the median RLN amplitude and latency of male and female patients in all three studies

\begin{tabular}{|c|c|c|c|c|c|c|c|}
\hline & & \multicolumn{3}{|c|}{ Median RLN amplitude/ $\mu \mathrm{V}$} & \multicolumn{3}{|c|}{ Median RLN latency/ms } \\
\hline & & Male & Female & $p$-value & Male & Female & $p$-value \\
\hline \multirow[t]{2}{*}{ Lorenz et al ${ }^{19}$} & Right & 478 & 837 & $<0.05$ & 2.73 & 2.73 & NS \\
\hline & Left & 501 & 772 & $<0.05$ & 2.73 & 2.73 & NS \\
\hline Phelan et $\mathrm{al}^{18}$ & - & $581^{a}$ & $739^{a}$ & NS & - & - & - \\
\hline \multirow[t]{2}{*}{ Our study } & Right & 900 & 940 & NS & 2.40 & 2.30 & NS \\
\hline & Left & 1190 & 990 & NS & 2.60 & 2.25 & 0.04 \\
\hline
\end{tabular}




aspect, Caragacianu et al, ${ }^{20}$ who had studied 125 consecutive thyroid surgeries with 167 RLNs at risk, found that the two injured RLNs had a statistically significant lower final mean amplitude (final mean amplitude of $97.5 \mu \mathrm{V}$ ) compared to their initial mean amplitude of $378 \mu \mathrm{V}$. This suggests that RLN nerve injury causes nerve amplitude to decrease. Finally, differences in technique of nerve stimulation and type of nerve monitoring systems may limit the generalizability of our results.

\section{CONCLUSION}

In this study of normative Asian EMG parameters for bilateral RLN stimulation, there was no significant difference between pre- and post- dissection RLN EMG parameters as well as between gender, nature of pathology and site of nerve. A prospective study needs to be carried out to investigate the effect of nerve injury on amplitude and latency.

\section{ACKNOWLEDGMENT}

Authors would like to thank Dr Yu Zhirong for his technical assistance with the inomed C2 NerveMonitorn $^{\circledR}$ (inomed Medizintechnik GmbH, Emmendingen, Germany).

\section{REFERENCES}

1. Kaplan E, Angelos P, Grogan RH. Surgery of the thyroid gland. In: De Groot LJ, Beck-Peccoz P, Chrousos G, (Eds). Endotext [Internet]. South Dartmouth (MA): MDText.com, Inc.; 2000.

2. Kaplan EL, Kadowaki MH, Schark C. Routine exposure of the recurrent laryngeal nerve is important during thyroidectomy. In: Simmons RL, Udekwu AO (Eds). Debates in Clinical Surgery, volume 1, Chicago USA: Yearbook;1990. p.191-206.

3. Chow TL, Chu W, Lim BH, Kwok SP. Outcomes and complications of thyroid surgery: retrospective study. Hong Kong Med J 2001 Sep;7(3):261-265.

4. Bergenfelz A, Jansson S, Kristoffersson A, Mårtensson H, Reihnér E, Wallin G, Lausen I. Complications to thyroid surgery: results as reported in a database from a multicenter audit comprising 3,660 patients. Langenbecks Arch Surg 2008 Sep;393(5):667-673.

5. Lydiatt DD. Medical malpractice and the thyroid gland. Head Neck 2003 Jun 25(6):429-431.

6. Kern K A. Medicolegal analysis of errors in diagnosis and treatment of surgical endocrine disease. Surgery 1993 Dec;114(6):1167-1173.

7. De Danschutter SJ, Schreinemakers JM, Smit LH van der Laan L, Nuytinck HK. Thyroid surgery and the usefulness of intraoperative neuromonitoring, a single center study. J Invest Surg 2015 Apr;28(2):86-94.

8. Dequanter D, Charara F, Shahla M, Lothaire P. Usefulness of neuromonitoring in thyroid surgery. Eur ArchOtorhinolaryngol 2015 Oct;272(10):3039-3043.

9. Pardal-Refoyo JL. Usefulness of neuromonitoring in thyroid surgery. Acta Otorrinolaringol Esp 2012 Sep-Oct; 63(5): 355-363.
10. Mangano A, Wu CW, Lianos GD, Kim HY, Chiang FY, Wang P, Xiaoli L, Hui S, Teksöz S, Bukey Y, et al. Evidence-based analysis on the clinical impact of intraoperative neuromonitoring in thyroid surgery: state of the art and future perspectives. Surg Technol Int 2014 Nov;25:91-96.

11. Randolph GW, Kamani D. Intraoperative neural monitoring in thyroid cancer surgery. Langenbecks Arch Surg 2014 Feb;399(2):199-207.

12. Pragacz K, Barczynski M. Evaluation of implementation phase of intraoperative neural monitoring of the recurrent laryngeal nerves in thyroid surgery in a district hospital. Przegl Lek 71(10):532-536.

13. Julien N, Mosnier I, Bozorg Grayeli A, Nys P, Ferrary E, Sterkers $\mathrm{O}$. Intraoperative laryngeal nerve monitoring during thyroidectomy and parathyroidectomy: A prospective study. Eur Ann Otorhinolaryngol Head Neck Dis 2012 Apr;129(2): 69-76.

14. Page C, Cuvelier P, Biet A, Strunski V. Value of intra-operative neuromonitoring of the recurrent laryngeal nerve in total thyroidectomy for benign goitre. J Laryngol Otol 2015 Jun;129(6), 553-557.

15. Cavicchi O, Caliceti U, Fernandez IJ, Macrì G, Di Lieto C, Marcantoni A, Ceroni AR, Piccin O. The value of neurostimulation and intraoperative nerve monitoring of inferior laryngeal nerve in thyroid surgery. Otolaryngol Head Neck Surg 2009 Jun;140(6):866-870.

16. Barczynski M, Konturek A, Cichon S. Value of the intraoperative neuromonitoring in surgery for thyroid cancer in identification and prognosis of function of the recurrent laryngeal nerves. Endokrynol Pol 2006 Jul-Aug;57(4):343-346.

17. Randolph GW, Dralle H, International Intraoperative Monitoring Study Group, Abdullah H, Barczynski M, Bellantone R, Brauckhoff M, Carnaille B, Cherenko S, Chiang FY, et al. Electrophysiologic recurrent laryngeal nerve monitoring during thyroid and parathyroid surgery: international standards guideline statement. Laryngoscope 2011 Jan;121 (Suppl 1):S1-16.

18. Phelan E, Potenza A, Slough C, Zurakowski D, Kamani D, Randolph G. Recurrent laryngeal nerve monitoring during thyroid surgery: normative vagal and recurrent laryngeal nerve electroal data. Otolaryngol Head Neck Surg 2012 Oct;147(4):640-646.

19. Lorenz K, Sekulla C, Schelle J, Schmeiss B, Brauckhoff M, Dralle H; German Neuromonitoring Study Group. What are normal quantitative parameters of intraoperative neuromonitoring (IONM) in thyroid surgery? Langenbecks Arch Surg 2010 Sep;395(7):901-909.

20. Caragacianu D, Kamani D, Randolph G W. Intraoperative monitoring: normative range associated with normal postoperative glottic function. Laryngoscope, 2013 Dec;123(12): 3026-3031.

21. Hermann M, Hellebart C, Freissmuth M. (Neuromonitoring in thyroid surgery: prospective evaluation of intraoperative electrophysiological responses for the prediction of recurrent laryngeal nerve injury. Ann Surg 2004 July; 240(1):9-17.

22. Lee HY, Cho YG, You JY, Choi BH, Kim JY, Wu CW, Chiang FY, Kim HY. Traction injury of the recurrent laryngeal nerve: Results of continuous intraoperative neuromonitoring in a swine model. Head Neck 2016 Apr;38(4):582-588.

23. Wu CW, Dionigi G, Sun H, Liu X, Kim HY, Hsiao PJ, Tsai KB, Chen HC, Chen HY, Chang PY, et al. Intraoperative neuromonitoring for the early detection and prevention of 
RLN traction injury in thyroid surgery: a porcine model. Surg 2014 Feb;155(2):329-339.

24. Poveda MCD, Dionigi G, Sitges-Serra A, Barczynski M, Angelos P, Dralle H, Phelan E, Randolph G. Intraoperative monitoring of the recurrent laryngeal nerve during thyroidectomy: a standardized approach (part 1). World J End Surg 2011;3(3):144-150.
25. Poveda MCD, Dionigi G, Sitges-Serra A, Barczynski M, Angelos P, Dralle H, Phelan E, Randolph G. Intraoperative monitoring of the recurrent laryngeal nerve during thyroidectomy: a standardized approach part 2. World J End Surg 2012;4(1):33-40.

26. Faden DL, Orloff LA, Ayeni T, Fink DS, Yung K. Stimulation threshold greatly affects the predictive value of intraoperative nerve monitoring. Laryngoscope.2015 May;125(5):1265-1270. 